\title{
Sachsen in neuer Gestalt Zur Verwaltungsgliederung Sachsens 1990 bis 2015
}

\author{
Jens Baumann
}

Bei der Abfassung des Aufsatzes griff der Autor auf eine eigene mehrjährige Untersuchung zurück. Vgl. Jens Baumann: Handlungsstrategien im Rahmen der Kreisgebietsreform - am Beispiel von Kreissitzbestimmung und Zentralitätsausgleich im Freistaat Sachsen. Dresden 2005; Jens Baumann: Der Neuzuschnitt der Landkreise in Sachsen - Anfang ohne Ende? In: Neues Archiv für sächsische Geschichte 78 (2007), S. 341-354
Land- und Stadtkreise in Sachsen 1945 bis 1952 (C) Andreas Oettel

\section{Herrsche und teile}

Dieses - wenngleich umgestellte - Bonmot führt uns verschiedene, sich reibende Aspekte der Raumbeherrschung vor Augen. Räume sind, neben wirtschaftlichen, sozialen, kulturellen und politischen Komponenten, rechtlich geprägt und administrativ gegliedert. Um Anspruchskonflikte beherrschen zu können, wurden Räume schon immer abgesichert: sei es militärisch, durch wirtschaftliche Garantien, politische Handlungsmuster, Bevölkerungsansiedlungen oder -verlagerungen. Gerade die Grenzziehungen sind verwaltungspolitisch bestimmt. Nach Søren Kierkegaard ist jede Grenze eine „wolllüstige Versuchung“, diese Grenze zu überschreiten, was illustriert, dass Grenzen keineswegs für die Ewigkeit gesetzt, sondern vielmehr Auslöser für grenzüberschreitende Aktivitäten sind, die diese Grenzen letztendlich selbst in Frage stellen. Werden Grenzen infrage gestellt, wird gleichzeitig auch Identität gefährdet, wie Christoph Waack ${ }^{1}$ nachwies, denn bei der Bil- dung von Nationalstaaten wurde „das Eigene, im Individuellen, wie im Kollektiv, abgeschlossen gegen das Fremde. Für die politischen Akteure ist damit ein Hauptziel ihrer Tätigkeit erreicht. Die territoriale Eindeutigkeit ihres politischen Machtbereichs und damit die auf diesem Gebiet lebende Bevölkerung stehen somit außer Frage. Damit wird ein Gefühl der Sicherheit produziert, das von der Bevölkerung nachgefragt wird. Ohne dieses agreement wäre eine nationalstaatliche Ordnung nicht denkbar."

Diese These wird im Folgenden vor allem auf die Ebene der Landkreisreform übertragen - mit der Fragestellung, ob eine Kreisbevölkerung als geistig-handlungsmotivierendes Konstrukt auftritt, um Besitzstände wie Kreisgebiet oder Kreisstadt zu verteidigen. Kreisstädte sind Symbol räumlicher Einheiten und beziehen die regionalen Aktivitäten politischer, wirtschaftlicher, verwaltungstechnischer und kultureller Art auf sich. Die Betonung kultureller Eigenheiten und die Suche nach

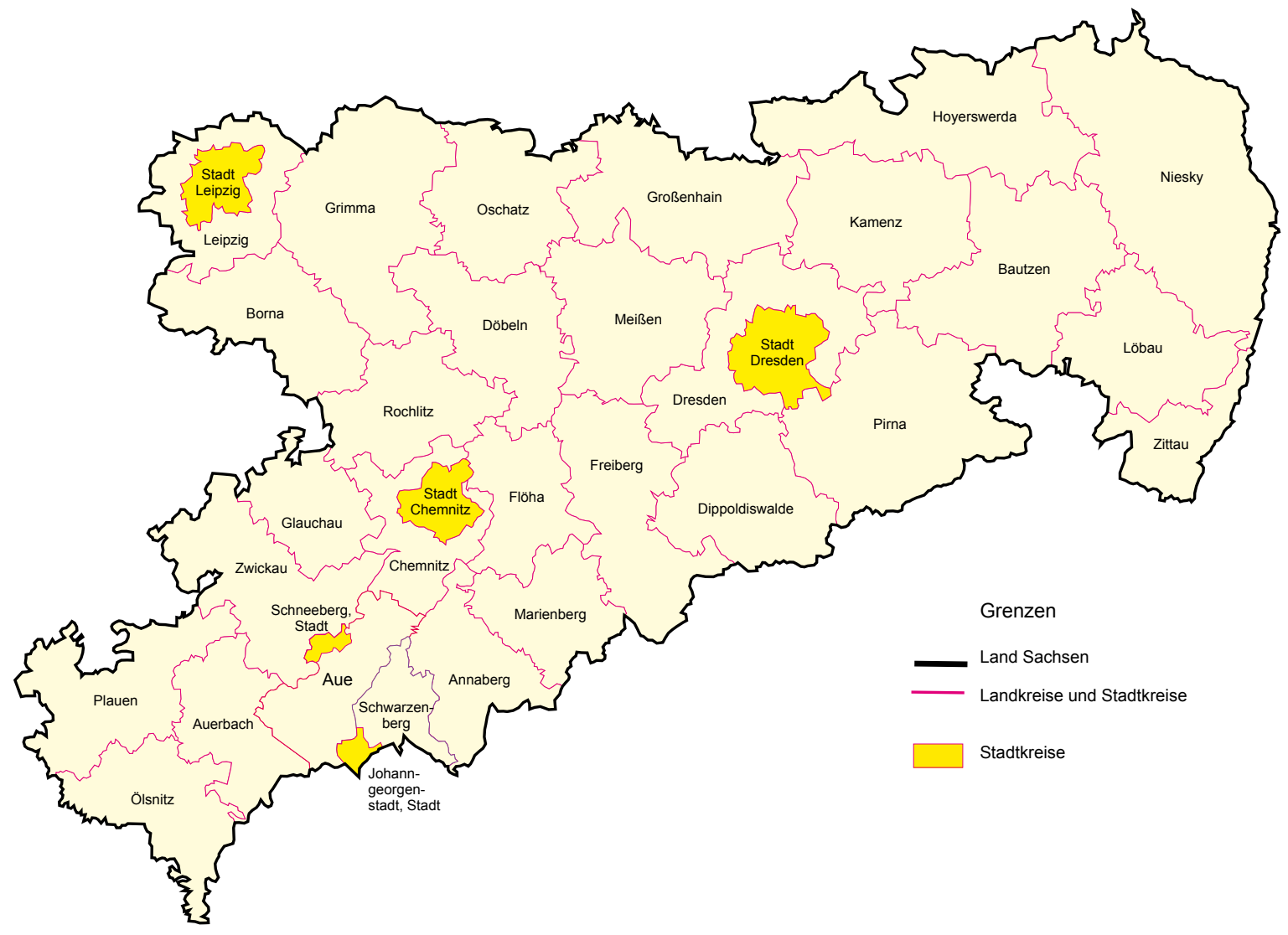




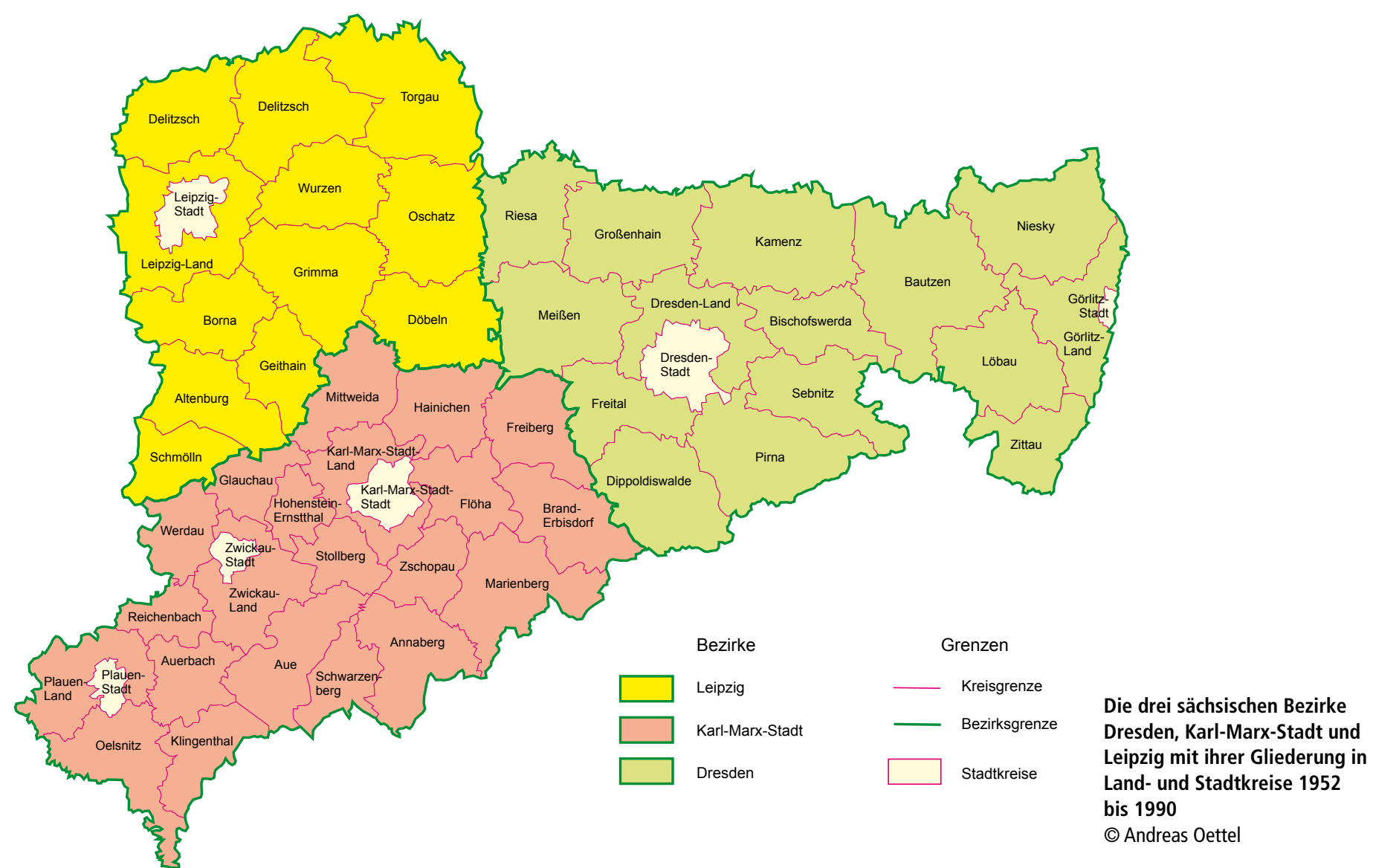

Identitäten lassen sich gleichsam als Gegenbewegung zur Globalisierung verstehen und werden gerade dort gestärkt, wo sie bislang als nicht notwendig erschienen. „Globalisierung“ ist mithin nur das Gegenstück von „Lokalisierung“.

Im Zuge der Herrschaftsverdichtung, der Ansammlung von Rechten und Besitz, von Titeln, Ämtern und Würden, kam es in Sachsen zu einer Gebietsverwaltung, die sich an historischen Räumen orientierte. Auf dem Gebiet des Kurfürstentums Sachsen gab es seit 1527 vier, seit 1553 sieben Kreise (jeweils mit mehreren Ämtern); daneben verschiedene Stifte und Herrschaften. Das spätere Königreich Sachsen teilte sich in Kreishauptmannschaften (Bautzen, Dresden, Chemnitz, Leipzig, Zwickau), die sich wiederum in Amtshauptmannschaften (z. B. Bautzen, Kamenz, Löbau, Zittau für die Kreishauptmannschaft Bautzen) untergliederten. Seit 1938 wurde für die bis dahin gültige Bezeichnung „Amtshauptmannschaft" nach preußischem Vorbild der Begriff „Landkreis“ oder „Kreis“ verwendet. Aus den „Kreishauptmannschaften“, den Mittelbehörden, wurden „Regierungsbezirke“. Die 29 Landkreise sowie bis zu acht Stadtkreise bestanden bis 1952.

Das vorgenannte Prinzip „herrsche und teile“ erfuhr dann in der DDR eine besondere Ausprägung: Die Vorbereitung der Länderauflösung über das „Gesetz über die weitere Demokratisierung des Aufbaus und der Arbeitsweise der staat- lichen Organe in den Ländern der DDR vom 23. Juli 1952“ führte zu einer Aufteilung der Arbeit der Landtage und Regierungen auf Bezirke und damit zu einer Mehrung und Verkleinerung der Verwaltungsgebiete bzw. einer funktionalen Aufwertung vor allem der mittleren Verwaltungsinstanzen. Gerade die Verkleinerung, bezüglich der Kreise in Sachsen fast Verdopplung der Verwaltungseinheiten überrascht, denn auch für die DDR hatten Fragen der Wirtschaftlichkeit und der Verfügung über ausreichende qualifizierte Arbeitskräfte einen hohen Stellenwert. Noch gewichtiger war aber eben das „teile“, nämlich die Erfordernis des „demokratischen Zentralismus“ respektive die dem SED-Staat unumgängliche Anleitung und Kontrolle. ${ }^{2}$ So spielten sicherheitspolitische, verkehrstechnische und geographische, kaum jedoch traditionelle Aspekte eine entscheidende Rolle. ${ }^{3}$ Gerade traditionelle Aspekte schienen ein Hindernis für eine sozialistische Arbeiter- und Bauernklasse zu sein. Historische Verwaltungssitze wurden durch neue Bezirksstädte ersetzt und so gleichzeitig „neue Zentren der Arbeiterklasse" geschaffen.

Was für die Bezirke galt, galt konsequenterweise auch für die Kreise: aus 121 Landkreisen wurden in der DDR 194, daneben erhöhte sich die Zahl der Stadtkreise von 22 auf 23. Für die drei Bezirke Dresden, Karl-Marx-Stadt und Leipzig formierte man 52 Land- und acht Stadtkreise, die bis zum
1 Christoph Waack: ,Regionen' an Staatsgrenzen und ,Grenzregionen. Ein Beitrag zur Diskussion konzeptioneller Elemente einer geographischen Grenzregionsforschung im östlichen Europa. In: Horst Fassel/Christoph Waack (Hrsg.): Regionen im östlichen Europa. Kontinuitäten, Zäsuren und Perspektiven. Tübingen 2000, S. 169-185.

2 Günter Püttner/Albrecht Rösler: Gemeinden und Gemeindereform in der ehemaligen DDR. Zur staatsrechtlichen Stellung und Aufgabenstruktur der DDR-Gemeinden seit Beginn der siebziger Jahre. Zugleich ein Beitrag zu den territorialen Veränderungen der Gemeinde- und Kreisgrenzen in der DDR. Baden-Baden 1997, S. 28.

3 Henning Mielke: Die Auflösung der Länder in der SBZ/DDR Von der deutschen Selbstverwaltung zum sozialistisch-zentralistischen Modell 1945-1952, Stuttgart 1995, S. 89; Frank Richter: Ökonomische Hintergründe der Verwaltungsreform von 1952 in der DDR. Dresden 1999, S. 26, räumt dem historischen Aspekt einen etwas höheren Stellenwert ein. 
Ende der DDR bestehen blieben. Eine Verwaltungskostensenkung - Grundprämisse jeder „modernen“ Gebietsreform - konnte also wohl kaum Ziel dieser Strukturreform gewesen sein. Henning Mielke erklärt die aus heutiger Sicht widersinnige Gliederung mit der absoluten „Gläubigkeit der SED-Spitze, dass sich mit der Durchsetzung des ,demokratischen Zentralismus' eine tiefgreifende qualitative Verbesserung der Verwaltung einstellen würde, vermittels derer ein größerer Apparat mit weniger Personal betrieben werden konnte." Folge dieser Gebietsreform der DDR war u. a., dass die Kreisstädte an Bedeutung bei der zentralen Planung, Investitionsvergabe und Versorgung gewannen; die Kreisbevölkerung identifizierte sich in diesen Fragen zunehmend mit ihrer Kreisstadt; ein Umstand, der nach 1990 zum Tragen kam und nicht zuletzt ein Zeichen dafür ist, in welchen Zeiträumen sich aus verordneter Identität eine selbstbewusste (ansatzweise) bilden kann.

Mit den Planungen zur Gebietsreform in Sachsen ab etwa 1991 mit dem Ziel einer Vergrößerung und damit Stärkung der Landkreise wurde Identität im Sinne von Bindung an den Verwaltungsstandort gefährdet. Somit trat auch der Zielkonflikt zwischen Effizienz und Integration hervor: Waren die Kommunen gerade doch erst durch die Kommunalwahlen 1990 in ihrer Selbstverwaltung und Identität aufgewertet worden, so sollten sie kurz darauf nach Effizienzgesichtspunkten vergrößert und also zusammengefasst werden. Dies musste als Bedrohung der eigenen Identität aufgefasst werden; Verlustängste, die an negativen Entwicklungsbedingungen festgemacht wurden, waren vorgezeichnet.

So war der Kampf um den Kreissitz erwartungsgemäß ein wesentliches Element vor allem der ersten Kreisgebietsreform auch in Sachsen. Die Bedeutung des Kreissitzes machte der damalige Vorsitzende der CDU-Landtagsfraktion wie folgt deutlich: „Bei den Streitpunkten - mit Ausnahme des Vogtlandes - geht es fast immer nur um die Frage des Kreissitzes. ${ }^{\text {4 }}$

\section{Die Kreisgebietsreformen im Freistaat Sachsen}

\section{Reform 1991 bis 1994}

Mit dem Verfassungsgesetz zur Bildung von Ländern in der Deutschen Demokratischen Republik vom 22. Juli 1990 wurden bereits durch die DDR zum 14. Oktober 1990 (durch den Einigungsvertrag vom 31. August 1990 wurde das Datum dann auf den 3. Oktober geändert) fünf Länder gebildet und die Bezirke abgeschafft. Das Land Sachsen entstand durch Zusammenlegung der Bezirksterritorien Dresden, Karl-Marx-Stadt/Chemnitz und Leipzig ohne die Kreise Altenburg und
Schmölln und zuzüglich der Kreise Hoyerswerda und Weißwasser. Freistaat wurde Sachsen erst seit dem 6. Juni 1992 durch das Inkrafttreten der im Sächsischen Landtag verabschiedeten Verfassung; Sachsen verbindet mit dieser Begriffswahl sehr alte staatliche Traditionen.

Bereits im Protokoll der 6. Sitzung des Sächsischen Landtages vom 13. Dezember 1990 tauchte der Gedanke einer notwendigen Gebietsreform auf dem Territorium des Freistaates Sachsen auf. Die Reformnotwendigkeit wurde im „Denkmodell des Sächsischen Staatsministeriums des Innern vom 14. November 1991“ mit dem landespolitischen Ziel einer modernen, leistungsfähigen und gestärkten kommunalen Selbstverwaltung begründet. Überwunden werden sollten insbesondere:

- die zu geringe Größe der Landkreise, denn fast die Hälfte aller Landkreise hatte weniger als 60.000 Einwohner - als Zielgröße galten fortan 125.000 Einwohner

- ihre zu geringe Leistungsfähigkeit, wobei Einwohnergröße und Leistungsfähigkeit weitgehend gleich gesetzt wurden

- eine Durchschneidung gleichartiger Siedlungs- und Wirtschaftsgebiete - der Ausbau einer öffentlichen Infrastruktur als Voraussetzung für wirtschaftliches Wachstum und die Lösung der Stadt-Umland-Problematik sollten insbesondere durch die Bildung von Sektoralkreisen gelingen; zugleich sollte zur Stärkung strukturschwacher Randgebiete der bisherige Kreissitz dort im Sinne des Prinzips der Solidarität im Raum verbleiben

- die Zerstörung gewachsener Gliederungen der traditionellen und bewährten Amtshauptmannschaften - die Kreisfreien Städte sollten eine Bestandgarantie erhalten, aber nicht zugleich auch Kreissitz werden.

Intendiert wurde damit, dass der dann größere Landkreis die bürgernah zu verwaltenden Aufgaben selbst bewältigen sollte, was nicht zuletzt als Voraussetzung für eine Funktionalreform im Sinne einer grundsätzlichen Aufgabenneuordnung zwischen kommunaler und staatlicher Ebene gesehen wurde. Doch obwohl an die traditionellen Amtshauptmannschaften gedanklich angeknüpft werden sollte, sollte gerade das Traditionsverständnis (Amtshauptmannschaft oder DDRKreisstadt), genährt durch das Machtpotential eines Landrates, zum Streitpunkt zu werden. War die Effizienz der Verwaltung, wie heute allgemein akzeptiert, ein wirkliches Anliegen, so konnte die argumentativ hervorgehobene Integrationsfunktion der neuen Landkreise, insbesondere natürlich der auszuwählenden Kreissitze, in den verschiedenen Positionsentwürfen hierfür nicht handlungsleitend sein. Die Infragestellung der 48
Protokoll der 70. Sitzung d Sächsischen Landtages, 25. Mai 1993. 
Landkreise, ihrer Kreissitze und der 6 Kreisfreien Städte kurz nach den Kommunalwahlen 1990 im Hinblick auf deren traditionelle Identifikationsrolle, vor allem vor dem Hintergrund des rasanten politischen, wirtschaftlichen und sozialen Wandels nach der deutschen Einheit, wurde nicht nur von der Kreisbevölkerung, teils von selbst teils animiert, sondern auch von den politischen Akteuren (gewählte Landräte und Kreistage) und den Verwaltungsmitarbeitern als Gefahr für ihre Machtposition bzw. für ihre Arbeitsplätze eingestuft.

Zum Verständnis der Konfliktbewältigung ist die von Paul Reuber formulierte Theorie raumbezogenen politischen Handelns interessant. ${ }^{5}$ Zentraler Bestandteil scheint ihm das sogenannte Konzept der Macht, wobei diese auf individuellen und institutionellen Komponenten (diese unterteilen sich in materielle und autoritative Ressourcen) beruht. Danach können sich Akteure immer dann entscheidend durchsetzen, wenn sie verschiedene wichtige Ressourcen vereinen (also z. B. Kreisvorsitzender einer Parteigliederung und Landrat). Zudem kommt die Selektivität der Wahrnehmung hinzu, wobei von besonderer Bedeutung der Entwurf strategischer Raumbilder durch die einzelnen Akteure ist. Mit ihnen werden räumliche Strukturen begründet, wobei ein und dieselbe Begründung je nach Akteursansicht für unterschiedliche Raumbilder respektive Kreissitze sprechen kann.

Wie spiegelte sich das nun im Kampf um die Kreissitzentscheidungen wider? Zum einen wurden durch die politischen Akteure und Verwaltungsspitzen, also diejenigen, die am ehesten Nachteile für ihre politische und berufliche Karriere befürchteten, die Bevölkerung z. B. zu Unterschriftensammlungen bzw. Volksanträgen umfassend mobilisiert (eigennutzerorientiertes Handeln). So wurde im Landkreis Flöha per Handzettel aufgefordert, seine Meinung direkt an das Innenministerium $\mathrm{zu}$ richten, wenn man nicht für „folgende Behördengänge nach Marienberg“ fahren wollte: „Kraftfahrzeugzulassung und -änderungen, Erlaubniswesen, Wohngeld, Lastenzuschüsse, Sozialhilfe, Arbeitslosenunterstützung, Behindertenbetreuung, Jugendunterstützung, Steuerangelegenheiten, alle Fördermittel und Zuschüsse, Regelung offener Vermögensfragen, Bauanträge und alle Grundstücksangelegenheiten und vieles andere mehr." Auffällig war dabei, dass solche Aktionen fast ausschließlich regional begrenzt liefen; bei Klärung der „Versorgungsfrage“ der politischen Amtsträger meist relativ schnell eingestellt wurden und oftmals entgegengesetzte Aktivitäten hervorriefen: so veranstaltete der Landkreis Marienberg gegen die Aktion der Flöhaer eine eigene Bürgerbefragung mit programmierten Ergebnis: „1. Sind Sie dafür, dass der Landkreis Marienberg in seiner Gesamtheit und mit dem Sitz der Kreisstadt Marienberg innerhalb des Mittleren Erzgebirgskreises bestehen bleibt?" Eine gewisse Autorität erlangten Protestbewegungen dann, wenn sie die Wendetradition der Montagsdemonstrationen aufnahmen; eine Strategie, die übrigens regelmäßig bis in die heutige Zeit angewandt wird: „Montagsdemonstrationen“ fanden so gegen das Kommunalabgabengesetz, gegen Schulschließungen und gegen den Sparkassenverband statt, auch Pegida hat sich den Montag zu eigen gemacht (ein Umstand, der, mit Blick auf das ursprünglich hohe demokratische Potential der Montagsdemonstrationen, besonders bei den Leipzigern breiten Missmut hervorrufen musste).

Der an strukturpolitischen Erwägungen orientierte Gesetzentwurf zur Neuordnung der Landkreise durch die Staatsregierung wurde aber weniger durch solche Bevölkerungsinitiativen geändert. Verfahrensleitend wurde vielmehr die „autoritativen Machtressourcen“ von zentralen Landtagsabgeordneten der ersten Wahlperiode, für die, nicht unbegründet, eine gleichzeitige Entscheidung zu Kreissitz und -gebiet und damit ein Interessensausgleich im Vordergrund stand. Dieser Ausgleich lief unter Moderation des CDU-Landtagsfraktionsvorsitzenden in sogenannten Konsensgesprächen als regionale Runden mit Landtagsabgeordneten, Landräten usw., auch um gleichzeitig mit den „,Verlierern' Verhandlungsangebote [zu erstellen], wie durch Ämtertausch bzw. Fördermöglichkeiten im Wohnungs- sowie Straßenbau der Verlust der Kreisstadtfunktion kompensiert werden kann. “ Eine entscheidungsdominante Rolle errangen damit die jeweiligen Wahlkreisabgeordneten bzw. solche mit einem hohen Sanktionspotential. So kam der CDU-Fraktionsvorsitzende aus der Kreisfreien Stadt Leipzig, die mit der ersten Kreisgebietsreform entgegen den oben genannten Grundsätzen den Kreissitzstatus erhielt. Sein Stellvertreter kam aus Marienberg; auch diese Stadt setzte sich gegenüber dem favorisierten Zschopau durch; das Mitglied des Landtages a. D. fand sich später in einer Führungsposition des Sächsischen Rechnungshofes wieder. Ähnliches lässt sich weiter mit Blick auf Mitglieder des Innen- sowie des Haushalts- und Finanzausschuss oder einzelner Minister prüfen: So war der Innenminister vorher Landrat des Landkreises Zittau, und Zittau wurde Kreisstadt des Landkreises Löbau-Zittau. Weitere autoritative Machtressourcen waren Positionen wie Landrat und Präsident des Sächsischen Landkreistages (dies war der Landrat des Landkreises Auerbach, der die Zweikreislösung für das Vogtland durchfocht) oder Landräte in Doppelfunktion als Landtagsabge-

\footnotetext{
5 Paul Reuber: Raumbezogene Politische Konflikte. Geographische Konfliktforschung am Beispiel von Gemeindegebietsreformen. Stuttgart 1999.
} 
ordnete, was in der ersten Wahlperiode noch zulässig gewesen war. Im Rückschluss konnten demzufolge diejenigen Kommunen bzw. Kreisstädte am ehesten punkten, die sich bei ihren Aktivitäten zur Wahrung des Kreissitzes insbesondere auf die Mobilisierung von CDU-Landtagsabgeordneten sowie des Kreisverbandes der Partei orientiert hatten.

$\mathrm{Zu}$ diesen autoritativen Machtressourcen traten die „materiellen Raumressourcen“: Statt strukturpolitischer Erwägungen wurden durch die autoritativen Machtressourcen nunmehr Zentralität und Größe der Kreissitze zu einem wichtigen Entscheidungsmoment für die Kreissitzbestimmung. Damit konnten sich vielfach große einwohnerstärkere Altlandkreise bzw. Kreisstädte und/oder zentrale Orte höherer Stufe durchsetzen, auch mittels selektiver Rauminterpretationen: So wurde beispielsweise die einwohnerschwächere Stadt Schwarzenberg als Kreissitz abgelehnt und Aue (Landrat in Doppelfunktion Landrat und Landtagsabgeordneter) gerade infolge seiner Zentralitätsfunktion sowie des Bevölkerungsschwerpunktes als Kreissitz bestimmt. Im Landkreis RiesaGroßenhain war es aber genau umgekehrt: hier sprach die strukturpolitische Komponente für die kleine Stadt Großenhain, während das große zentrale Riesa den Kreissitz verlor. Und die Kreissitzentscheidung für Zittau berief sich auf deren „periphere Lage im Dreiländereck ... [die] einer

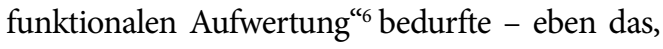
was dem peripheren Schwarzenberg gerade verwehrt wurde. Prämissen wie "Solidarität im Raum“ und „keine Kreisfreie Stadt als Kreissitz“, so die Fälle Leipzig und Görlitz, traten damit zurück.

\section{Reform 1995 bis 1998}

Dieses Umsteuern und eine gewisse Nichtsystematik in den Entscheidungsbegründungen riefen selbstverständlich die Reformverlierer auf den Plan, die Verfassungsklagen einreichten. Erfolg zeitigten diese aber im Rückblick nur dann, wenn sie sich auf Form- und Verfahrensfehler, insbesondere bzgl. einer mangelhaften Anhörung, stützen konnten; in den eigentlichen räumlichen Entscheidungen und deren Begründungen ließen die Verfassungsrichter dem Gesetzgeber seinen ihm gebührendem Rang. So trat die erste Kreisgebietsreform am 1. August 1994 nur teilweise in Kraft. Dies machte drei Kreisgebietsreformänderungsgesetze notwendig. Das „Erste Gesetz zur Änderung des Kreisgebietsreformgesetzes und anderer kommunalrechtlicher Vorschriften vom 6. September 1995" führte zum 1. Januar 1996 Teile des Landkreises Dresden-Land mit dem Landkreis Meißen zum neuen Kreis Meißen-Radebeul (ein Jahr später Umbenennung in Meißen) zusam- men. Zudem wurden zum selben Zeitpunkt ein Landkreis Westlausitz-Dresdner Land (ein Jahr später Umbenennung in Kamenz; hierzu gehörten nun Gemeinden aus den ehemaligen Landkreisen Bischofswerda, Dresden-Land, Hoyerswerda und Kamenz) und Hoyerswerda als Kreisfreie Stadt gebildet.

Mit dem „Zweiten Gesetz zur Änderung des Kreisgebietsreformgesetzes vom 6. September 1995 “wurde das Vogtlandgebiet als nur ein Landkreis zum 1. Januar 1996, nämlich als Vogtlandkreis, geschaffen; die ehemals im Kreisgebietsreformgesetz vorgesehenen Landkreise Elstertalkreis und Göltzschtalkreis kamen gar nicht erst zustande. Der Vogtlandkreis umfasst alle bisherigen Landkreise: Auerbach, Klingenthal, Oelsnitz, Plauen-Land und Reichenbach.

Durch das „Dritte Gesetz zur Änderung des Kreisgebietsreformgesetzes vom 23. Mai 1996“ wurde für den Niederschlesischen Oberlausitzkreis ab dem 16. Juni 1996 der Kreissitz von der Kreisfreien Stadt Görlitz in die kreisangehörige Stadt Niesky verlegt. Hier ist die wechselhafte Begründung interessant: Sprachen ehemals die Zentralitätsfunktion des Oberzentrums sowie damit verbundene Entwicklungsimpulse für Görlitz, so galt nun, dass ,auch unter Berücksichtigung der von der Stadt Görlitz wahrzunehmenden zentralen Funktionen und der sich hieraus ergebenden Wirkung auf das umliegende Territorium diese Wirkung von der Kreissitzfestlegung nicht berührt [wird]. Die zentralen Funktionen der Stadt Görlitz bleiben erhalten, so dass die Kreissitzverlegung keinen Wertverlust in Bezug auf die räumliche Standortbewertung der Stadt Görlitz und ihr Umland zur Folge hat. Die Möglichkeit des Einkaufens im Zusammenhang mit der Erledigung von Behördengängen spielt angesichts der zunehmenden Aufgabenverlagerung auf die Gemeindeebene und der verhältnismäßig geringen Anzahl der Bürgerkontakte mit der Kreisverwaltung nur eine untergeordnete Rolle. Die Kreisfreie Stadt Görlitz ist aufgrund ihrer räumlichen Lage ein natürlich gewachsenes Zentrum des Verflechtungsbereiches Niederschlesien. Die daraus abzuleitende versorgungsräumliche und kulturelle Ausstrahlung sowie die aus der Grenzlage resultierenden planungspolitischen Entwicklungsperspektiven der Stadt Görlitz wirken sich auf deren Gesamtentwicklung relativ positiv aus, so dass eine zusätzliche ,Anstoßfunktion, wie sie mit der Kreissitzbestimmung verfolgt wird, nicht zwingend erforderlich ist. Eine fehlende Akzeptanz der Kreissitzentscheidung bei erheblichen Teilen der Bevölkerung kann sich auf Dauer nachteilig auf die notwendige Integration und die zu wahrende örtliche Verbundenheit der Einwohner auswirken. [Im Übrigen hat sich] die 
überwiegende Mehrheit der Bevölkerung des Niederschlesischen Oberlausitzkreises bereits bei der Anhörung zu der Entwurfsfassung des Innenausschusses im Januar 1993 gegen einen Kreissitz Görlitz ausgesprochen. "7 So wurde aus der ehemaligen Mehrheit für Görlitz in der ersten Kreisreform (Niesky und Görlitz gegen Weißwasser) nun die Mehrheit für Niesky.

Ebenso wurde im Umkreis der Kreisfreien Stadt Leipzig mit dem „Gesetz zur Regelung der Stadt-Umland-Verhältnisse im Bereich der Kreisfreien Stadt Leipzig vom 24. August 1998“ der Kreissitz von Leipzig an Borna zum 1. Januar 1999 gegeben.

Als Anmerkung sei noch darauf verwiesen, dass mit dem Landkreis Döbeln offensichtlich niemand etwas anzufangen wusste, der vorerst einfach fortbestehen blieb.

\section{Reform 2006 bis 2008 und Konsolidierung}

Ende 2006/Anfang 2007 wurde eine erneute, wohl nunmehr abschließende, Kreisgebietsreform - die mit einer umfassenden Verwaltungsreform verbunden sein sollte ${ }^{8}$ - eingeleitet, die zum 1 . August 2008 in Kraft trat. Überraschend war, dass schon fünf Jahre nach dem vollständigen Abschluss der ersten Reform und praktisch im Anschluss an die damals nachfolgende Gemeindegebietsreform erneute Gedanken einer Reformfortführung auftraten. Dies zeigt vor allem, dass die vorangegangene Kreisreform gerade nicht die Voraussetzungen für eine umfassende Funktionalreform im Sinne einer Aufgabenneuverteilung zwischen Land und Kommunen erfüllte und die gebildeten Einheiten von den beschließenden Gremien selbst nicht als zukunftssicher eingeschätzt wurden. Eine derart rasche Aufeinanderfolge von eher langfristig angelegten Reformen verunsichert nicht zuletzt sowohl die Bürger als auch die Verwaltung selbst, von den erneuten Kostenfaktoren sowie der Frage der Bewertung der Amortisierung der bereits eingebrachten Kosten ganz zu schweigen. Damit steht ein wesentliches Moment der Berechenbarkeit von staatlichem Handeln, einer Säule unseres funktionierenden Staatswesens, vielleicht irgendwann in Frage.

In der Begründung des Gesetzentwurfes waren wiederum der enge Zusammenhang zu einer nachfolgend/parallel erfolgenden Aufgabenübertragung auf die kommunale Ebene sowie die Kosteneinsparung von vorrangiger Bedeutung, wenn auch offensichtlich nicht ermittelbar: „Die Kreisneugliederung wird auf Dauer die Leistungsfähigkeit der Landkreise verbessern. Die Vergrößerung ermöglicht es, das Leistungspotenzial zusammenzufassen, konzentriert einzusetzen und so die Wirkung zu steigern. Neben qualitativen Verbesserungen sind auch Kostenreduzierungen reali- sierbar. In jedem Falle ist zu erwarten, dass sich in absoluten Zahlen nach der Konsolidierung der Verhältnisse in den neuen Landkreisen durch die Nutzung von Synergie- und Skalierungseffekten eine freie Spitze im Gesamthaushalt ergibt. In welcher Größenordnung die Maßstabsvergrößerung zu Einsparungen bei den Personal- und Sachkosten führt, ist im Einzelfall nur schwer einzuschätzen und hängt wesentlich davon $a b$, wie die Landkreise in Ausgestaltung ihrer kommunalen Selbstverwaltung die durch die Kreisneugliederung geschaffenen Einsparpotentiale ${ }^{9}$ ausnutzen." Insbesondere galten nun als Einwohnermindestgröße 200.000 Einwohner für die Prognose 2020. Die Zielgröße hatte sich damit von ehemals 125.000 Einwohnern binnen ca. 14 Jahren um das 1,6-fache erhöht. Großkreise, also z. B. Landkreise in Form der Kulturräume, sollten aber weiterhin vermieden werden. Weitere Gesichtspunkte waren infrastrukturelle, kulturelle, historische und andere Gegebenheiten; ein wichtiges Kriterium war zudem auch die Berücksichtigung der Stadt-Umland-Problematik. Allerdings reformierten verschiedene staatliche Verwaltungen wie die Kultusverwaltung ihren nachgeordneten Bereich selbst und unabhängig vom Entwurf des Innenministeriums, ebenso wurde die Mittelebene nicht grundsätzlich angetastet; aus den Regierungspräsidien wurden zum 1. August 2008 die Landesdirektionen, seit dem 1. März 2012 nur noch die Landesdirektion Sachsen mit Sitz in Chemnitz (jedoch unverändert mit den drei selben Standorten).

Allein schon den Pressemeldungen war zu entnehmen, dass die größten Streitpunkte die Kreissitze waren, außerdem standen für die öffentliche Diskussion die Kreisnamen und die KfzKennzeichen im Vordergrund. Die meisten Spannungen respektive Kämpfe bestanden zwischen den „gesetzten“ Verlierern Grimma, Kamenz und Aue sowie den „Siegern“ Borna, Bautzen und Annaberg-Buchholz. Daneben verloren vier von sieben kreisfreien Städten ihre Kreisfreiheit (Aufsichtsbehörde wurde damit das Landratsamt, so für die bisherige kreisfreie Stadt Hoyerswerda nun das Landratsamt Bautzen), denen u. a. als Äquivalent der Status einer Großen Kreisstadt, Sonderregelungen im Kulturraumgesetz sowie in drei Fällen, Görlitz, Zwickau und Plauen, auch der Kreissitz zuerkannt wurde. Fraglich erscheint aber so im Nachhinein die erst 1995 beschlossene Aufwertung von Hoyerswerda zur Kreisfreien Stadt, die nun, aufgrund des offensichtlich nicht vorhersehbaren Bevölkerungsschwundes, diesen Status nicht nur verlor, sondern es nicht einmal mehr zu einer Kreisstadt schaffte. Dies lässt breiten Interpretationsspiel-
6 Protokoll der 41. Sitzung des Innenausschusses vom 6 . bis 8 Mai 1993.

7 Drucksache 2/0420 Sächsischer Landtag, Beschlussempfehlung und Bericht.

8 Vgl. Wolf-Uwe Sponer: Gesamtkonzept für eine Funktional- und Verwaltungsreform im Freistaat Sachsen. In: Landesund Kommunalverwaltung 8 (2006), S. 337-347.

9 Gesetz zur Neugliederung des Gebietes der Landkreise des Freistaates Sachsen und zur Änderung anderer Gesetze (Referentenentwurf), Begründung Punkt 8. Demgegenüber stehen die tatsächlichen Transformationskosten, vgl. den Zentralitätsverlustausgleich in Höhe von über 28 Mio. Euro (Plenarprotokoll 4/125 vom 9. Dezember 2008) und die Anpassungshilfe für alle betroffenen Landkreise und Kreisfreien Städte in Höhe von 260 Mio. Euro (Gesetz zur Neugliederung des Gebietes der Landkreise, $\$ 26$ ), um allein be belastbaren Zahlen zu bleiben. 
Verwaltungseinheiten im Freistaat Sachsen 1994/96 bis 2008 und seit 2008 (c) Andreas Oettel raum für die Sinnhaftigkeit der ehemaligen Statusaufwertung.

Signifikant dafür, dass nicht nur die beiden Prozesse Kreisgebietsreform 1994 und 2008 vergleichbar sind, sondern auch die maßgebenden Handlungsstrategien, ist das Beispiel des Kreissitzstreites zwischen Grimma und Borna. Grundvoraussetzung eines jeden Kreissitzentscheides ist der Zuschnitt des zukünftigen Landkreises. Erst durch die Zuordnung des Landkreises Döbeln nach Freiberg/Mittweida geriet der ehedem fest geglaubte Kreissitz Grimma ins Wanken, da die deutliche Mittelpunktlage verloren ging. Hinzu kam selektiv die Strukturschwäche als Argument für Borna; mit dem gleichen Argument hatte Borna in der ersten Kreisreform 1994 gerade nicht durchdringen konnte und verlor den Kreissitz an Leipzig. Allerdings wurde dem Argument der Strukturschwäche im Gesetzentwurf gerade sonst nicht Rechnung getragen, denn alle anderen vorgeschlagenen Kreissitze zielen auf die historisch bedeutendere und auch sonst lage- und ausstattungsmäßig gewichtigere Stadt, insofern musste sich Grimma als Gegenpart zu Borna getäuscht sehen. Nicht zuletzt wog das „Konzept der Macht“ schwer. Der bis dahin existierende Landkreis Leipziger Land mit der Kreisstadt Borna war der einzige Landkreis, der von einer Landrätin der SPD geführt wurde. Insofern ist es nicht abwegig anzunehmen, dass mit Rücksicht auf die damalige Große Koalition hier zumindest auch weitere Gesichtspunkte eine Rolle spielten. Auch die anderen beschriebenen Strategien finden sich wieder: Gedroht wurde mit Parteiaustritten ebenso wie mit dem Ruhen der Mitgliedschaft (Oberbürgermeister der Kreisfreien Stadt Zwickau), Gemeinden wurden mit Geld vom Nachbarlandkreis zu einem Kreiswechsel gelockt und um anstelle von Annaberg doch noch Kreissitz zu werden, strebte Aue eine Zeit lang die Vereinigung mit Lößnitz, Schneeberg und Bad Schlema zur neuen Stadt Silberberg mit dann ca. 50.000 Einwohnern an.

Nach rund 15 Jahren Kreisgebietsreform in Sachsen ergibt sich folgender Überblick:

1. Mit der ersten Kreisgebietsreform in Sachsen, unter Einschluss ihrer Änderungsgesetze, verringerte sich die Zahl der Landkreise von 48 auf 22, die Anzahl der kreisfreien Städte wuchs hingegen von sechs auf sieben (Hoyerswerda).

2. Mit der zweiten Kreisgebietsreform verringerte sich die Anzahl der Landkreise nochmals, nun auf nur noch zehn. Ebenso fielen vier von sieben kreisfreien Städten weg.

3. Kreisstädte sind heute Bautzen und Görlitz in der Oberlausitz, Plauen für das Vogtland, Annaberg für den Erzgebirgskreis, Freiberg für Mittelsachsen, Borna für den Landkreis Leipzig, Torgau für Nordsachsen, Pirna für die Region Sächsische Schweiz/Osterzgebirge, Meißen für

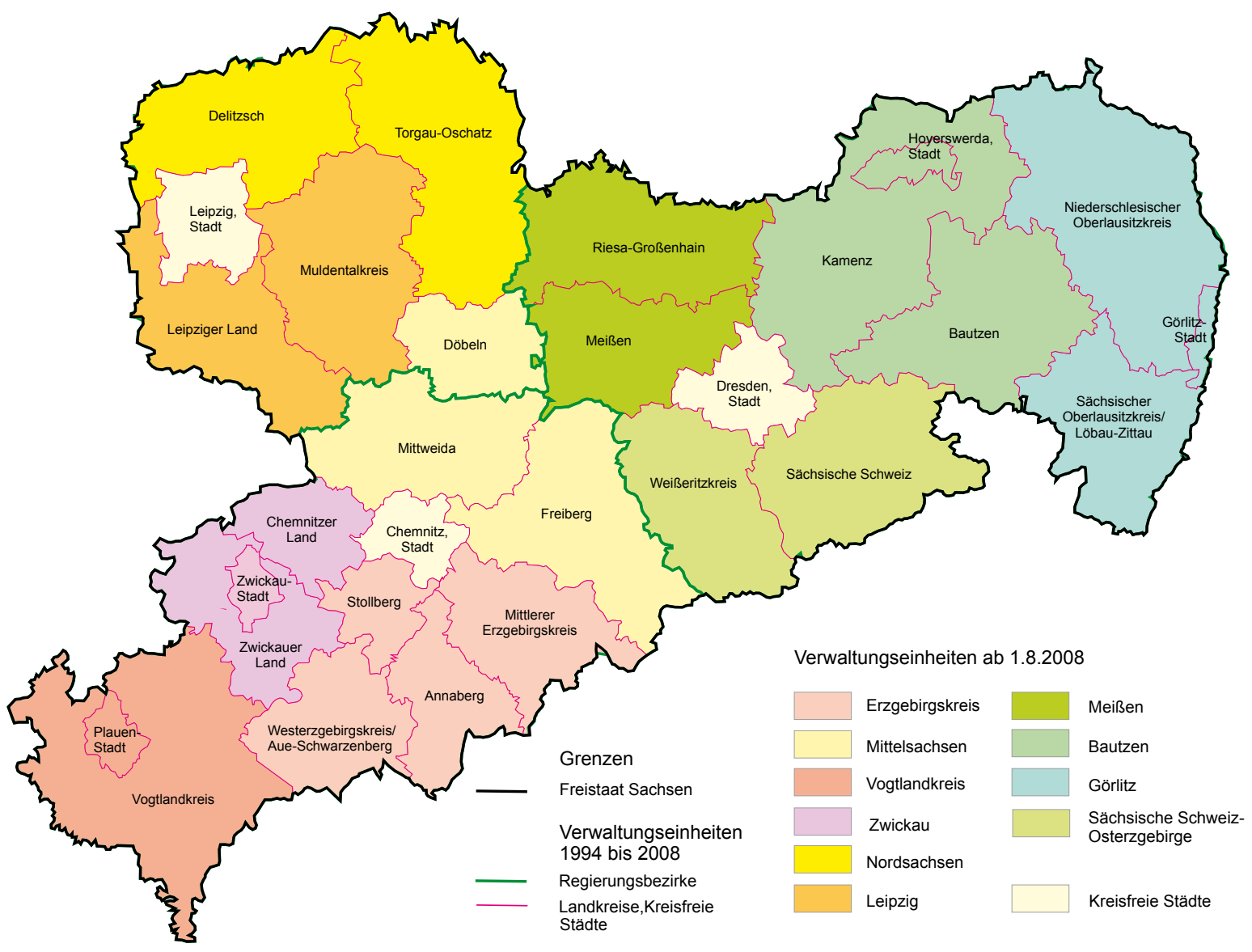


den Bereich Meißen/Riesa/Großenhain und Zwickau für den Bereich Chemnitzer Land/ Zwickauer Land. Damit sind drei ehemalige kreisfreie Städte zumindest Kreissitz geblieben, was ihre zentralörtliche Funktion herausstellt (Görlitz, Plauen, Zwickau). Kreisfreie Städte sind selbst keine Kreisstädte, ein schon von der Staatsregierung seit 1991 verfolgtes Ziel.

4. Mehrfache Kreissitzwechsel blieben nicht aus, so die Entscheidungen im Leipziger Raum (Borna versus Leipzig) und in der Oberlausitz: hier war von August 1994 bis Juni 1996 Görlitz Kreissitz, dann Niesky und nun seit dem 1. August 2008 wieder Görlitz. Auch der kreisfreien Stadt Hoyerswerda war vom 1. Januar 1996 bis zum 1. August 2008 nur ein relativ kurzes Leben beschieden.

5. Die Einwohnerzielgrößen wurden mit durchschnittlich rund 275.000 Einwohnern pro Landkreis sicher erreicht (Bezug 2013).

\section{Auswirkungen des Kreissitzverlustes}

Nachdem in den vorangegangenen Abschnitten das Zustandekommen der heute abschließenden Kreissitze nachgezeichnet wurde, bleibt die Frage nach den Folgen des Kreissitzverlustes, die ja permanent als negativ für die zukünftige Exkreisstadt vermutet wurden. Als entscheidend prognostizierten nach der ersten Kreisgebietsreform und den diesbezüglichen Kreisgebietsreformänderungsgesetzen die durch den Autor befragten Bürger- und Oberbürgermeister sowie Landräte in 2001/2002 dabei den kommunalen und staatlichen Behördenabzug, den mangelnden Neubau infrastruktureller Kreiseinrichtungen und einen signifikanten Bevölkerungsrückgang. Diese Vermutungen waren nicht unbegründet. Für den kommunalen und staatlichen Behördenabzug wurden die Verlagerungen von Kreissparkasse, Kreisvolkshochschule etc. geprüft. Im Ergebnis hatten bis ins Jahr 2000 in den untersuchten zwölf Landkreisen die verbleibenden Kreisstädte insgesamt 16 Einrichtungen gegenüber 1994 verloren, die Exkreisstädte im gleichen Zeitraum aber 46 Einrichtungen. Auch der staatliche Behördenabbau betraf die Exkreisstädte überdurchschnittlich. Nach ausgewählten Behördenzweigen ${ }^{10}$ verloren diese insgesamt 45 Behörden, die Kreisstädte nur 30. Bedenkt man dann die Beschäftigtenquote im öffentlichen Dienst, die sich durch den neuen Raumzuschnitt ergibt, so lassen sich Raumveränderungen erkennen, die zu Benachteiligungen in strukturschwachen Gebieten geführt haben. ${ }^{11}$ Daraus resultierte ein verändertes kommunales Ausgabeverhalten der im öffentlichen Dienst Beschäftigten, die ja über relativ sichere, qualifizierte und sachgemäß bezahlte Arbeitsplätze verfügen, das negative Folgen für die weitere kommunale Entwicklung zeitigen kann.
Zudem war zu erkennen, dass sich Investitionsentscheidungen hin zum neuen Kreissitz ergeben haben. Ebenso ließ sich ein überdurchschnittlicher Bevölkerungsrückgang der Exkreisstädte konstatieren, der für den Zeitraum von 1990 bis 2000 14,8 Prozent gegenüber 12,1 Prozent bei den Kreisstädten betrug. Im Ergebnis dieser Untersuchungen lassen sich eine Zentralitätseinbuße der Kreissitzverlierer und ein Zentralitätszuwachs für die Kreissitzgewinner durchaus bestätigen. So waren alle Kreisstädte nach dem Landesentwicklungsplan 2003 mindestens als Mittelzentren eingestuft worden, wobei die Ausweisung als Mittelzentren natürlich wiederum die Kreissitzfunktion in Rechnung stellte. Exkreisstädte erfuhren hingegen zum Teil eine Herabstufung.

Die staatlichen Ausgleichsmaßnahmen konnten hier nicht entscheidend entgegenwirken, da kommunale und staatliche Strategien nicht durchweg konform liefen. Wichtige Handlungsmöglichkeiten ergaben sich durch umfangreiche Ausgleichszahlungen (insgesamt knapp 134 Millionen DM 1994 ff., dann 2008 ff. knapp 300 Millionen Euro), die Verleihung der Rechtsstellung Große Kreisstadt, die Einrichtung von Außenstellen der Landratsämter und staatliche Behördenverlagerungen (sofern diese durchgeführt wurden), während sich weitere Maßnahmen wie eine besondere Berücksichtigung bei Wirtschaftsförderprogrammen oder bei der nachfolgenden Gemeindegebietsreform (etwa durch Eingemeindungen) als nahezu wirkungslos erwiesen.

\section{Gemeindegebietsreform}

Bei der Gemeindegebietsreform war eine Verfünffachung des ehemaligen Zieles (Eigenständigkeit ab 1.000 Einwohnern; nunmehr ab 5.000) zu beobachten. Bemerkenswert ist, dass dies ganz ohne formales Gesetz, also auf untergesetzlicher Ebene, ablief. Seit 1994 gab es Grundsätze für eine kommunale Zielplanung im Freistaat Sachsen, die dann
10 Vermessungsämter, Amtsgerichte, Schulämter, Finanzämter, Staatliche Ämter für Ländliche Neuordnung, Staatliche Ämter für Landwirtschaft und Gartenbau, Staatliche Forstämter.

11 Die Exkreisstädte wiesen 1992 im staatlichen Behördenbesatz 48,6 Beschäftigte/1000 Einwohner gegenüber 63,4 in den Kreis städten auf. Dies änderte sich im Gefolge der Kreisreform auf 24,1 Beschäftigte/1000 Einwohner gegenüber 59,7 in den Kreisstädten.

Das neue Landratsamt des Landkreises Görlitz in der Görlitzer Bahnhofstraße Foto: Lars-Arne Dannenberg

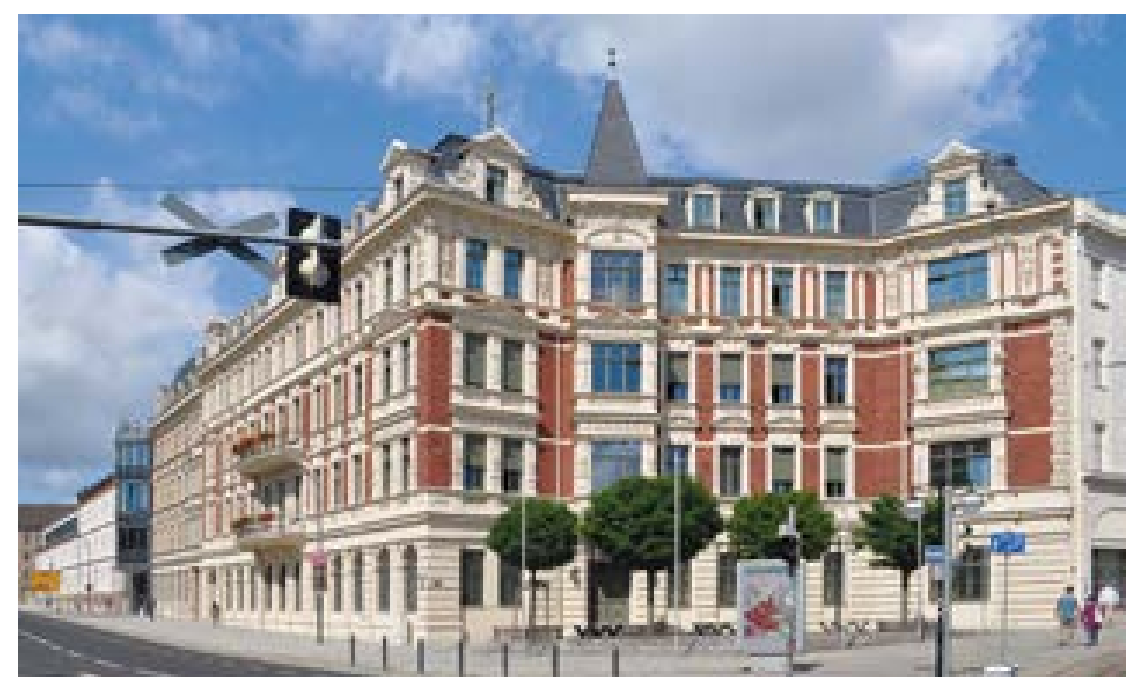




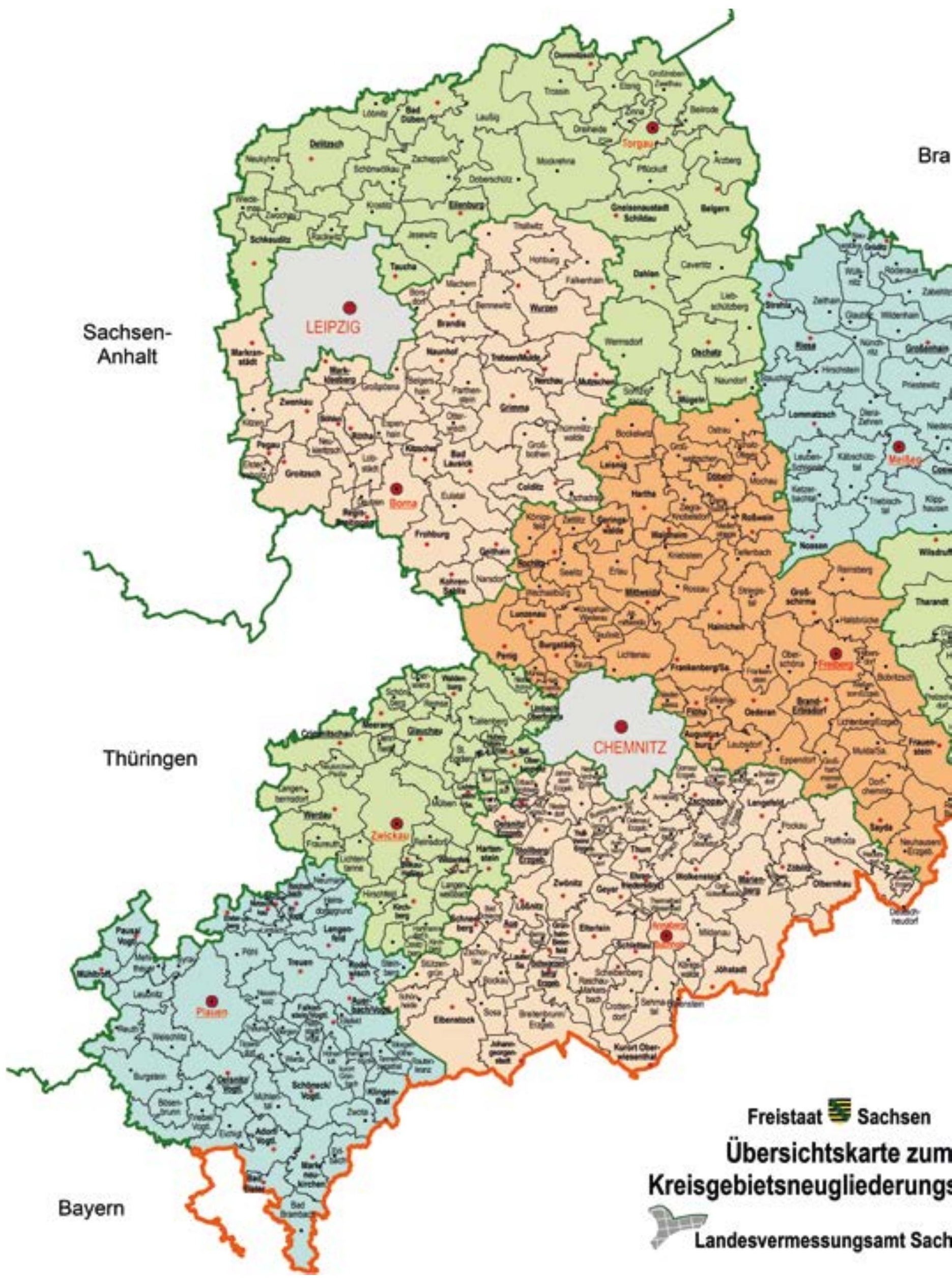


ndenburg

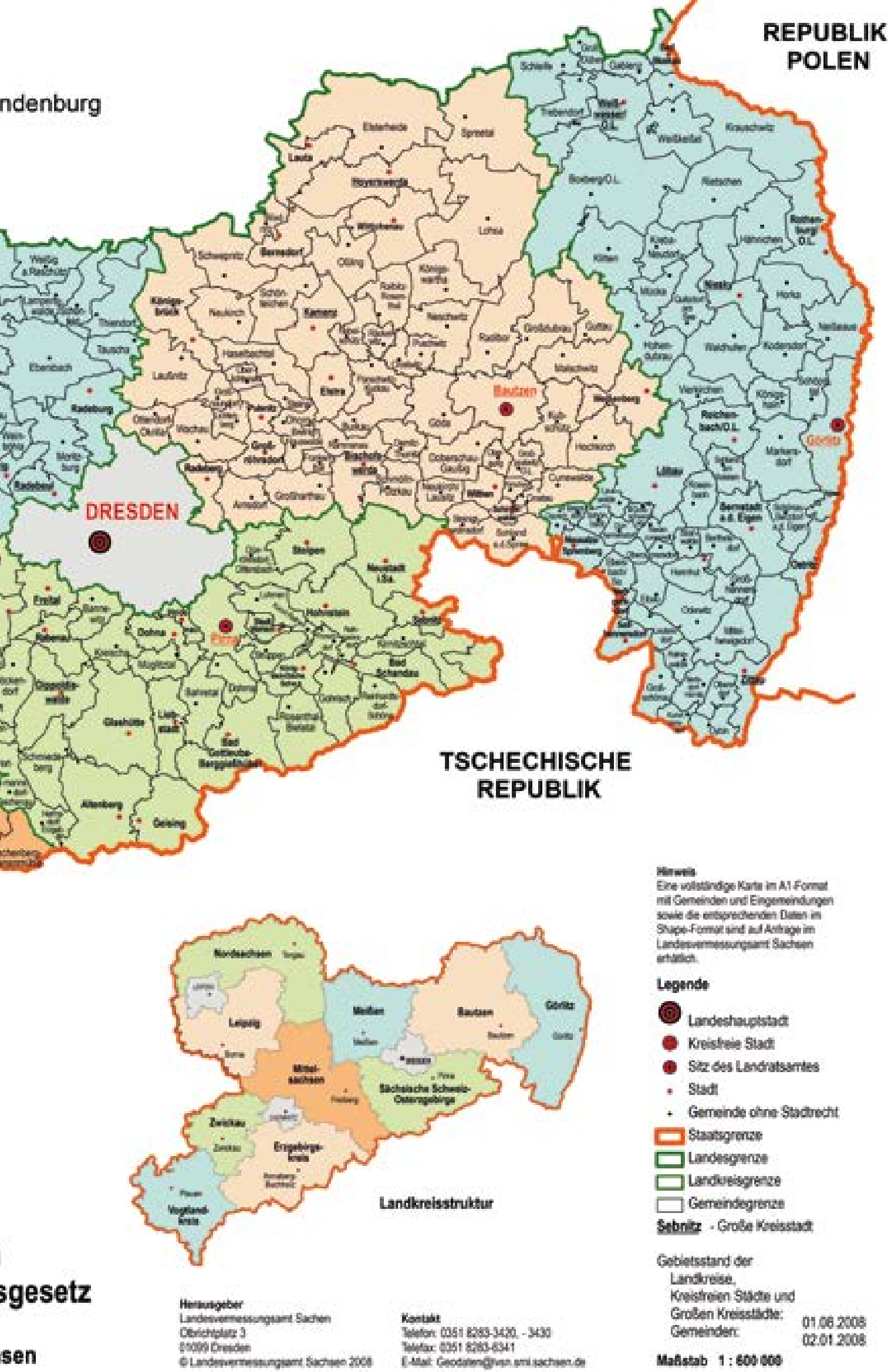

Landkreise und Gemeinden im Freistaat Sachsen, Stand 2008 (C) Landesvermessungsamt Sachsen 


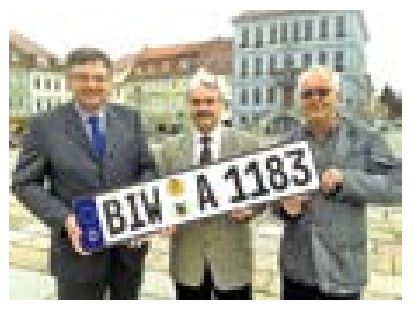

Der Identitätskonflikt auf Kreisebene und das Verlustbewusstsein entzündete sich nach 2008 an den KfZ-Kennzeichen. Holger Zastrow (links), damals Vorsitzender der sächsischen FDP, mit dem wiedereingeführten Auto-Kennzeichen BIW für Bischofswerda, 2012 Foto: FDP-Ortsverband Bischofswerda

12 SächsABl. 2010 Nr. 45, S. 1620.

13 SächsABl. 2014 Nr. 13, S. 523, vormals Verwaltungsvorschrift des Sächsischen Staatsministeriums des Innern zu kommunalen Namen ( $\mathrm{VwV}$ kommunale Namen) vom 27. Januar 1995 (SächsABl. S. 256).

14 Vgl. im Übrigen Jens Baumann: Sächsische Gemeindenamen im Wandel, in: Mitteilungen des Landesvereins Sächsischer Heimatschutz 3/1995, S. 55-59.

15 Quelle Statistisches Jahrbuch Sachsen sowie eigene Berechnungen.

16 Quelle Statistisches Jahrbuch Sachsen sowie eigene Berechnungen. später in Grundsätze für freiwillige Zusammenschlüsse im Freistaat Sachsen in Form lediglich einer Verwaltungsvorschriff ${ }^{12}$ weitergeführt worden - im Vorgehen und in den Ergebnissen eine durchaus beachtenswerte Leistung. Hauptetappen der Gemeindereform waren die Jahre 1992 und dann 1995, 1998 sowie nochmals nach 2007, also einmal vor und sonst nach den Kreisgebietsreformen, um gerade bzw. auch gerade nicht durch Gemeindezusammenschlüsse vor der Festlegungen der Landkreisgrenzen bestimmte Landkreisbildungen zu präjudizieren; die Kreisgebietsreform hatte Vorrang. Zwangseingemeindungen gibt es keine, aber dennoch in jedem Jahr Eingliederungen, Zusammenlegungen oder Gebietsaustausch zwischen den Gemeinden.

Die in den 1990er Jahren geschaffenen Verwaltungsverbände sterben durch Eingliederungen und keine Neugenehmigungen langsam aus. Auch hier spielte und spielt natürlich die Frage der Leistungskraft der Gemeinde und ein ausgewogenes Verhältnis zwischen Einwohnern und Verwaltungsmitarbeitern eine wichtige wenn nicht die zentrale Rolle, also der stille finanzielle Druck (bessere Schuldenlastverteilung bei größeren Einheiten usw.). So wird in der Verwaltungsvorschrift eine „zunehmende Diskrepanz zwischen der (schwindenden) Einwohnerzahl der Gemeinden und den (steigenden) Aufwendungen zur Aufrechterhaltung der Daseinsvorsorge" gesehen. Nicht zuletzt sehen auch die Kommunen, ihre Räte, Mitarbeiter und Bürgermeister mit gemischten Gefühlen auf das baldige Auslaufen des Solidarpaktes und die sich daraus ergebenden Folgerungen für die weitere Finanzierung kommunaler Aufgaben. Die immer höheren europäischen Anforderungen an beispielsweise Baumaßnahmen und Umweltstandards sowie die in Sachsen mittlerweile eingeführte Doppik gegenüber der kameralen Haushaltsführung machen den Ernst der Lage zur Reduzierung von Verwaltungsaufgaben, um über freie Mittel weiterhin verfügen $\mathrm{zu}$ können, nur noch deutlicher. Investitionen sind fast nur noch über Förderprogramme darstellbar, wozu es aber einer Eigenkapitalquote bedarf. Neben der Reduzierung von freiwilligen Aufgaben und der Gewinnung von bürgerschaftlichen Engagement für bestimmte thematische Gemeindevorhaben bleibt eben oft nur der Ansatz der Reduzierung von Verwaltungskosten, also der Abbau von Personal. Dies kann dann auch zu neuen Gemeindezuschnitten führen.

Wichtig war den Kommunen insbesondere auch der Gemeindename. Dieser steht außer Frage bei Eingliederungen. Hier kann und wird der Name der eingegliederten Gemeinde als Ortsteilname, ggf. auch manchmal als Ortschaftsname (wenn es einen eigenen Ortschaftsrat gibt) erhalten, während die aufnehmende Gemeinde den neuen
Gemeindenamen für das neue Gesamtgebilde gibt. Schließen sich aber in etwa gleich große Gemeinden zusammen, so bestand oft der Wunsch nach einem neuen Gemeindenamen, um nicht als Sieger respektive als Verlierer "namentlich“ dazustehen. Nun besitzt Sachsen aber ein reiches historisches Erbe, welches sich eben auch in den überkommenen Namen widerspiegelt. Auch hier konnte mit einer Verwaltungsvorschrift ${ }^{13}$ viel gerettet werden und unsinnige Namensvorschläge abgewehrt werden; zu denken sei an Höhe 9, Apfelland, Zum Wasserturm usw. Vielfach wurde auf Gewässernamen (Triebischtal, Ketzerbachtal, Elsterheide) oder Berg-, Burg-, Flurnamen (Burgstein, Kriebstein, Amtsberg) Bezug genommen. Schön ist es im Übrigen, dass Sachsen sich auch wieder die Verleihung von Bezeichnungen zu-traut. So darf sich Freiberg seit 2015 nun Universitätsstadt Freiberg nennen; ein Titel, der noch in den 1990er Jahren vom damaligen Finanzminister Georg Milbradt auf eine Initiative des Innenministeriums hin abgelehnt worden war, um einer dauerhaften Besitzstandswahrung (Titel und damit Universität) mit Blick auf finanzielle Rahmenbedingungen vermeintlich vorzubeugen. Bezeichnungen wie Pfefferkuchenstadt (für Pulsnitz) oder Bergstadt und dergleichen sind hingegen keine offiziellen Titel oder Namensbestandteile. ${ }^{14}$

\section{Was bleibt?}

Die Zeit großer Gebietsreformen scheint nunmehr abgeschlossen. Eine weitere Vergrößerung respektive Zusammenlegung auf beispielsweise nur noch fünf Landkreise würde zum einen wohl die Existenzberechtigung einer Landesdirektion, zumindest aber von zwei der drei Standorte selbst in Frage stellen, bedingte also eine wirkliche Funktionalreform (Neuverteilung der Aufgabenwahrnehmung zwischen staatlicher und kommunaler Ebene). Auch die Frage, was meint Bürgernähe, wäre dann wirklich zu diskutieren. Zudem würde das Gleichgewicht zwischen den dann sehr mächtigen (im Sinne der von ihnen wahrzunehmenden Aufgaben) Landräten und der Staatsregierung neu zu tarieren sein. Auch bei den Gemeinden gibt es nur noch marginale Änderungen hinsichtlich der Gemeindezahlen, also nur noch wenige Zusammenschlüsse bzw. Eingliederungen.

Interessant ist die Entwicklung der Größenverhältnisse. Sachsen hat hier konsequent, wenn auch gegen Widerstände, auf eine Reduzierung kleiner kommunaler Einheiten zugunsten relativ selbständiger kommunaler Körperschaften hingearbeitet, die sich auch wirtschaftlich behaupten können. Insofern erscheint der Weg als richtig. Auch aus der Presse heraus ist ein zunehmendes Abebben der Widerstände sichtbar; die Bevölkerung hat 


\begin{tabular}{|l|l|l|l|l|}
\hline & $\begin{array}{l}\text { Anzahl der Kreise/ } \\
\text { Kreisfreien Städte }\end{array}$ & $\begin{array}{l}\text { Bevölkerung } \\
\text { (durchschnittlich) }\end{array}$ & $\begin{array}{l}\text { Kleinste Bevölke- } \\
\text { rungszahl / größte } \\
\text { Bevölkerungszahl }\end{array}$ & $\begin{array}{l}\text { Größe in } \\
\text { Quadratkilometer } \\
\text { (durchschnittlich) }\end{array}$ \\
\hline $\mathbf{1 9 9 1}$ & $48 / 6$ & 65.608 & $22.361 / 118.755$ & 369 \\
\hline $\mathbf{1 9 9 5}$ & $22 / 7$ & 139.238 & $81.555 / 234.881$ & 802 \\
\hline $\mathbf{2 0 1 4}$ & $10 / 3$ & 274.205 & $197.346 / 351.309$ & 1.757 \\
\hline
\end{tabular}

sich mit den Gegebenheiten arrangiert, Ortschaftsräte und mobile Verwaltungseinrichtungen gerade im ländlichen Raum wirken hier positiv. Vergleichend sei auf Brandenburg und Thüringen verwiesen. Brandenburg wies 2011 vier kreisfreie Städte, 14 Landkreise und sowie 419 Gemeinden auf. Dies ergibt eine durchschnittliche Landkreisgröße von 151.000 Einwohnern sowie durchschnittlich 5.100 Einwohner pro Gemeinde ohne Kreisfreie Städte. Thüringen hingegen gliederte sich 2011 in 17 Landkreise, 6 kreisfreie Städte (am wenigsten Einwohner hat Suhl mit ca. 35.000 Einwohnern) und 913 Gemeinden. Dies ergibt eine durchschnittliche Landkreisgröße von 100.000 Einwohnern sowie durchschnittlich 1.880 Einwohner pro Gemeinde ohne Kreisfreie Städte.

Im Übrigen lassen sich zwei grundsätzliche Schlussfolgerungen ziehen:

Erstens: Gebietsreformen resultieren aus territorialen Verhältnissen, die in Verfassungsverhältnissen der Zeitepoche gründen. Sich ändernde Verfassungsverhältnisse wie der Gewinn der kommunalen Selbstverwaltung sowie zunehmende Unsteuerbarkeit und Inflexibilität wirken destabilisierend und begründen damit Überlegungen $\mathrm{zu}$ einer Verwaltungsreform (die sich aber nicht auf gebietliche Maßnahmen beschränken muss). Zentrales Begründungsmuster ist immer wieder das Verhältnis von Einwohnerzahl und Leistungskraft und damit die Suche nach einem optimalen Zahlwert zur weitgehenden Selbstverwaltung. Die Neuschneidung von Landkreis- oder Gemeindegrenzen und damit Änderungen des Verwaltungssitzes lösen politisch-räumliche Konflikte aus, wenn sich die regionalen Akteure mit dem überkommenen Kreisgebiet und der Kreisstadt identifizieren. Bestehen keine verbindlichen politischen Entscheidungskriterien, können diese Konflikte unter Zuhilfenahme von eigennutzerorientiertem Handeln, individuellen, materiellen und autoritativen Macht- und Raumressourcen sowie subjektiven strategischen Raumbildern gelöst werden. Eine wesentliche Motivation der handelnden politischen Akteure ist dabei der Erhalt oder die Vergrößerung ihrer Machtbasis (dies gilt gerade auch räumlich), wobei es diese Akteure vielfach verstehen, den Identitätswunsch und das Identitätsstreben lokaler Bürgergesellschaften zu nutzen. Folglich spiegeln die Festlegungen von Verwaltungsgrenzen und -sitzen auch das Handeln der Akteure wider.
Zweitens: Die Neugliederung kann eine Verschlechterung der Standortqualität der Exkreisstädte bewirken und weitere Verwaltungsentscheidungen wie Standort- und Ausweisungsfragen negativ beeinflussen. Verwaltungsentscheidungen konstituieren damit neue Räume und neue funktionsräumliche Beziehungen, die auf die neue Kreisstadt orientiert sind. Dies zieht Folgen für die Erreichbarkeit/Verwaltungsleistungen, die Investitionsvergabe, den Behördenabbau beziehungsweise die Behördenansiedlung nach sich. Die regionalen Akteure empfanden den Kreissitzverlust als umfassenden Verlust, und eine Standortumbewertung tritt auch faktisch ein. Eine Gegensteuerung durch eine moderierende Regionalplanung unter Einbeziehung aller kommunalen Akteure auf Basis landesplanerischer Eckwerte ist somit nötig.

Es bleibt festzuhalten, dass hinreichend große Einheiten mit einer regionalen Selbstbestimmung und eigenen Identität am ehesten langfristigen Reformwünschen und auch kommunalen Perspektiven ent-

\begin{tabular}{|l|l|l|}
\hline Jahr & Gemeindeanzahl & $\begin{array}{l}\text { Einwohner durchschnittlich pro } \\
\text { Gemeinde ohne kreisfreie Städte }\end{array}$ \\
\hline 1991 & 1.623 & 1.948 \\
1992 & 1614 & \\
$1993 / 1994$ & 970 & 3.226 \\
1995 & 860 & 3.591 \\
1996 & 809 & \\
1997 & 787 & \\
1998 & 564 & 5.484 \\
1999 & 544 & \\
2003 & 522 & \\
2007 & 496 & 5.998 \\
2008 & 493 & \\
2013 & 432 & 6.392 \\
\hline
\end{tabular}

sprechen. Weiterhin erleichtern kommunal orientierte und angemessene Ausgleichsprozesse die Neubildung von Gebietseinheiten. Politische Prämissen bestimmen die Reformen immer mit, gerade deshalb erscheint es umso wichtiger, diese zu kennen. Ein Patentrezept, wie Verwaltungssitze bestimmt werden sollen, welche Verluste für die negativ betreffenden Städte eintreten und wie diese sicher auszugleichen sind, kann der Autor nicht vorschlagen, und es bleibt auch nach Sichtung der überaus reichhaltigen Literatur fraglich, ob dies jemals beweisfest gelingen kann.
Entwicklung der Gemeinden in Sachsen ${ }^{16}$

Dr. Jens Baumann

Sächsisches Staats-

ministerium des Innern Wilhelm-Buck-Straße 2 01097 Dresden

\section{Autor}

Entwicklung der Landkreise in Sachsen ${ }^{15}$ 